\title{
A Novel Model for the Evolution of Online Public Opinion Based on Fuzzy Cellular Automata Rules in Directed World Networks
}

\author{
Tao Chen ${ }^{1,2}$ and Jie Lin ${ }^{1, *}$ \\ ${ }^{1}$ School of Economics and Management, Tongji University, Shanghai, China \\ ${ }^{2}$ Department of Information Management, School of Business, Ningbo University, Zhejiang, China
}

Received: 16 Mar. 2013, Revised: 19 Jul. 2013, Accepted: 20 Jul. 2013

Published online: 1 Nov. 2013

\begin{abstract}
In this paper, a model of Cellular Automata model (CA), which contains four essential characters composed of Emotional tendencies of current netizen, self-confidence, the impact and sentiment orientation from other netizens, was designed for the evolution of online public opinion. To establish the relationship between the state of current netizen and the impact rate from other netizens. This study has adopted the rule-based fuzzy system. With the support of some experts from the Psychology area, 70 fuzzy rules with different weight have been formulated. Through the analysis of the simulation results, the opinion concentration, polarization, are revealed. The results of simulation also show that all four essential characters have a certain influence on individual behavior as well as group's, and the changing character of neighbors will influence individual behavior. Simulation results also indicate that the model can better fit with the public opinion evolution laws.
\end{abstract}

Keywords: Evolution, online public opinion, Fuzzy cellular automata, directed small-world networks.

\section{Introduction}

Nowadays, with the fast development of Web 2.0 technology, the web is its melting pot. More and more people are willing to write blog articles to express their personal feelings, emotions and attitudes on their daily activities and hot topics on the Internet. According to the reports from CNNIC (China Internet Network Information Center), by January 2012, the number of Chinese blog has reached 319 million, and it is reaching 62 percent of the total Internet users in China (http://www.cnnic.net.cn) [1]. Internet becomes "free market" where the netizen can freely express any opinion, Internet makes peoples thought and speech freely, enrich and stimulate. Online public opinion can be defined as people's knowledge, attitude, emotional or behavioral tendencies through the Internet which due to stimulation arising from any events.

Online public opinion can be defined as the collection of opinions of many different people on the Web and the sum of all their views [2]. Governments have increasingly found public opinion to be useful tools for guiding their public information and propaganda programs and occasionally for helping in the formulation of other kinds of policies. For individual users, public opinion can help them when making decisions.

Most recently, research on online public opinion focus on opinion mining, opinion formation and evolution, opinion mining techniques have been used to find peoples attitudes in blogosphere. Previous studies on opinion retrieval in blogs usually focus on finding the topic relevant opinionated blog entries [3, 4], but not the opinion relatedness between the retrieval results. The existing studies on opinion summarization can generate a short abstract of the majority opinions in a close blog dataset on a given topic [5]. However, for public opinion monitoring task, the extracted results should not only contain the summary of opinions, but also should include the formation and evolution of opinion. Opinion formation and evolution models are divided into two classes, discrete opinion and continuous opinion models. In discrete models each agent has only one of a finite

\footnotetext{
*Corresponding author e-mail: linjie@ tongji.edu.cn
} 
number of different opinions, so these models are suitable to describe some situations, for instance, a binary choice in a political election. In this group, the Ising model considers both the influences of neighbors and additional global magnetization, observing the phenomena of phase transition [6]. The majority rule model in which agents adopt the local majority opinion of their neighbors seems factual $[7,8]$. In the other group continuous opinion models, which mostly use the concept of bounded confidence, allow opinions to be extreme. Deffuant put forward a model where agents change opinions towards each other splitting the difference [9, 10], and Hegselmann-Krause premised an individual would take the average value of opinions of nearby agents [11]. The methods mentioned above all use the theory of the small league affecting the people around as their basic idea. Alves investigates the influence in the process of Brazils general election [12]; Weisbuch investigates the influence of decision-making time on ideology in Ref. [13]. Some of above take the cellular automata model as the basic influence rule.

Fuzzy set approaches were developed for special purposes where the information basis was vague and imprecise [14], especially for online public opinion. Under these conditions fuzzy techniques allow concise conclusions in contrast to the other approaches, which cannot be employed successfully because of a lack of data. In this paper, this study emphasize the evolution of online public opinion under the fuzzy rules. A precondition were taken that different environments have different influences on people, and consider every individual as having the ability to influence others. It is also considered that the smaller the characteristic distinction between individuals is, the more the influence there is; and the minority can be easily assimilated by the majority. With these definitions, this study develop the fuzzy cellular automata model of the evolution of public sentiment.

\section{Fuzzy Cellular Automata rules in small network}

\subsection{Cellular Automata}

The Cellular Automata (CA) was introduced in the 1950s by the mathematician John von Neumann, who considered the suggestions from Stanislaw Ulam and tried to model the natural process of self-reproduction [15]. and was extended to a broader scientific field by Wolfram in 1986 [16]. It is a class of spatially and temporally discrete mathematical system characterized by local interactions and an inherently parallel form of evolution. CA are discrete dynamical systems formed by a set of identical objects called cells. These cells are endowed with a state which changes at every discrete step of time according to a deterministic rule.

In short, the CA consists in discrete simulations in time, space and the system state. The idea of these models consists in considering each position (or region) of the spatial dominion as being a cell which is attributed to a state. The state of each cell is modified regarding both its own state and its neighbor's during the former time stage, through a series of simple rules that try to imitate the physical or biological laws, which are, in our case, the ones that rule the system. In this approach, the system state variables, as well as the time, are discrete.

One of the most important $\mathrm{CA}$ is two-dimensional finite CA. More precisely, a two-dimensional finite CA can be defined as a 4-uplet $A=(C, S, V, f)$, where $\mathrm{C}$ is the cellular space formed by a two-dimensional array of $l \times s$ identical objects called cells: $C=\{\langle i, j\rangle, 0 \leq i \leq l-1,0 \leq j \leq s-1\}$, such that each of them can assume a state. The state of each cell is an element of a finite or infinite state set, $\mathrm{S}$; if $\mathrm{S}$ is finite and $|S|=k$ then $S$ is taken to be $Z_{k}=\{0,1, \cdots, k-1\}$. The state of the cell $\langle i, j\rangle$ at time $\mathrm{t}$ is denoted by $a_{i j}^{(t)}$. The set of indices of the 2D-CA is the ordered finite subset $V \subset Z \times Z,|V|=M$, such that for every cell $\langle i, j\rangle$, its neighborhood $V_{i j}$ is the ordered set of m cells given by $V_{i j}=\left\{\left\langle i+\alpha_{1}, j+\beta_{1}\right\rangle, \cdots,\left\langle i+\alpha_{m}, j+\beta_{m}\right\rangle:\left(\alpha_{k}, \beta_{k}\right) \in V\right\}$ .There are some classic types of neighborhoods, but in this work only the extended Moore neighborhood will be considered. That is ,more neighborhood with radius $r=1$ of the cell is $\langle i, j\rangle$ given by the following set of indices: $V_{M}=\{(-1,-1),(-1,0),(-1,1),(0,-1),(0,0),(0,1)$, $(1,-1),(1,0),(1,1)\}$.

The 2D-CA evolves deterministically in discrete time steps, changing the states of all cells according to a local transition function $f: S^{8} \rightarrow S$. The updated state of the cell $\langle i, j\rangle$ depends on the nine variables of the local transition function, which are the previous states of the cells constituting its neighborhood ( if $r=1$ ), that is:

$$
a_{i j}^{(t+1)}=f\left(a_{i+\alpha_{1}, j+\beta_{1}}^{(t)}, \cdots, a_{i+\alpha_{8}, j+\beta_{8}}^{(t)}\right)
$$

The matrix

$$
C^{(t)}=\left(\begin{array}{cccc}
a_{00}^{(t)} & a_{01}^{(t)} & \cdots & a_{0, s-1}^{(t)} \\
a_{10}^{(t)} & a_{11}^{(t)} & \cdots & a_{1, s-1}^{(t)} \\
\vdots & \vdots & \ddots & \vdots \\
a_{l-1,0}^{(t)} & a_{l-1,1}^{(t)} & \cdots & a_{l-1, s-1}^{(t)}
\end{array}\right)
$$

is called the configuration at time $\mathrm{t}$ of the 2D-CA, and $C^{(0)}$ is the initial configuration of the CA. Moreover, the sequence $\left\{C^{(t)}\right\}_{0 \leq t \leq k}$ is called the evolution of order $\mathrm{k}$ of the 2D-CA.

The detailed CA modeling procedure as may be completed by the following steps. 
S1. The objective space is partitioned into discrete cells of regular (square lattice or hexagonal lattice) or irregular lattice with finite volumes. The square lattice is chosen in this paper, and this study will discuss the different evolutionary result with the moore neighborhood with various radius $r$.

S2. Usually several state values are assigned to each cell. These values are called cell states which determine the spatial and temporal evolution of the complex system .

S3. At each time step, a local neighborhood is defined for each cell.

S4. The switching rules (function $\mathrm{f}$ ) are defined as a function of its previous state and the state of the neighboring sites. In this work, function $\mathrm{f}$ is a fuzzy function.

After the completion of definition of above steps, CA simulation proceeds by updating state variables of each cell through the local switching rules owing to these characteristics. Elementary cellular automata have been studied for their ability to generate complex global behavior, despite their simplicity. One variation of it is obtained by adding memory to each cell in a neighborhood. This process generates a provisional configuration in which the application of an evolution rule establishes the dynamics of the system [17]. Some researchers extensively studied in the fuzzy domain, and gave a probabilistic interpretation between the Boolean and the fuzzy models [18].

\subsection{Fuzzy rule-based systems}

Fuzzy logic was first developed by Zadeh (1965) in the mid-1960s for representing uncertain and imprecise knowledge [19]. It provides an approximate but effective means of describing the behavior of system that are too complex, ill-defined, or not easily analyzed mathematically. The literature on uncertainty has grown considerably during these last years, especially in the areas of system modeling, optimization, control, and pattern recognition. Recently, several authors have advocated the use of fuzzy set theory to address epidemiology problems [20-22] and population dynamics [23].

A fuzzy subset A of the a universal set $X$ is defined by a membership function $\mu_{A}$ that assigns to each element $x$ of $X$ a number $\mu_{A}(x)$, between 0 and 1 , which gives by degree of membership from $x$ to A. Thus, $\mu_{A}: X \rightarrow[0,1]$. It interesting to note that a classic subset $\mathrm{A}$ of $X$ is a particular fuzzy set for which the membership function is the its characteristic function of A, $x_{A}: X \rightarrow\{0,1\}$.

A concept that will play a key role in this paper is Fuzzy rule-based systems, whose structure is shown in Fig.1 [25]. Fuzzy rule-based systems (FRBS) have four components: an input processor, a collection of fuzzy rules called rule base, a fuzzy inference machine, and an output processor. These components process real-valued inputs to provide real-valued outputs as follows:

- Input processor (Fuzzification). Here, inputs are translated into fuzzy sets of their respective universes. For numerical inputs, the most commonly used approach transforms a real value into a fuzzy singleton. In the general case, expert knowledge plays an important role to build the membership functions for each fuzzy set associated with the inputs.

- Rule base. This is a key, knowledge encoding component of fuzzy rule based systems. It is composed by a collection of fuzzy conditional propositions in the form if-then rules. Fuzzy rules provide an effective means to encode expert knowledge expressed in the form of linguistic statements. Essentially, fuzzy rules are fuzzy relations of the Cartesian product of the universes of the variables of interest. This is a knowledge-encoding component of fuzzy rule-based systems, a collection of fuzzy conditional propositions in the form of If-then rules. Fuzzy rules are an effective mean to encode expert knowledge expressed through linguistic statements.

- Fuzzy inference. The fuzzy inference machine performs approximate reasoning using the compositional rule of inference. A particular form of fuzzy inference of interest here is the Mamdani method.

- Output Processor (Defuzzification). In fuzzy rule-based systems, the output usually is a fuzzy set. Often, especially in system modeling, the system require a real number as output.The output processor is the one who provides real outputs through defuzzification,a process used to choose a real number that is representativeof the corresponding fuzzy set. A typical defuzzification scheme, the same as adopted in this paper, is the center of gravity method defined as follows. Let $C$ be the membership function of the output variable $z$. Then the real-valued output $\bar{z}$ is chosen as follows:

$$
\bar{Z}=\frac{\int z C(z) d z}{\int C(z) d z}
$$

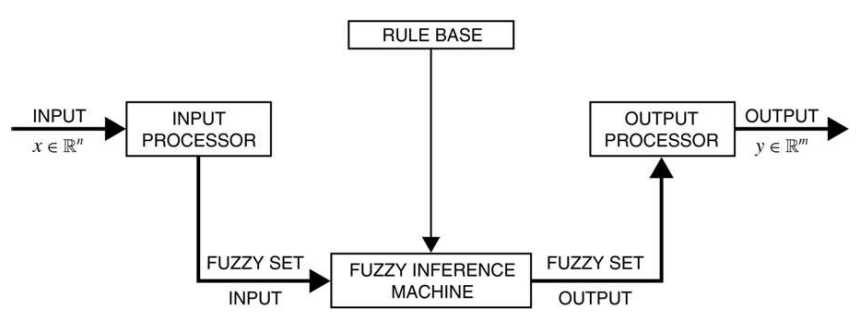

Fig. 1: Structure of fuzzy rule-based systems [25]

Fuzzy cellular automata (FCA) are continuous cellular automata where the local rule is defined as the "fuzzification" of the local rule of a corresponding 
Boolean cellular automaton in disjunctive normal form. The integrative application of Fuzzy cellular automata (FCA) has popularized in many fields and has displayed a wide prospect. Such as, geographical information science, environment planning and design, Knowledge-Based System, etc [24-27]. In the following section, this study proceed to introduce the fuzzy model, the main aim of this paper.

\subsection{Directed Small-world rule}

The small-world model of undirected networks was originally proposed by Watts and Strogatz [28]. The WattsCStrogatz model [29] is one of the simplest network models exhibiting the small-world effect which allows to smoothly change from a regular to a random lattice. In our model, small world networks are adopted in order to represent real social networks. Small-world networks have highly local clustering (i.e. neighboring nodes are connected tightly) and short average paths (i.e. one node is only a few paths away from any other node in the network), thereby supporting the coexistence of segregation and integration.

The two-dimensional network is made of $\mathrm{N}$ identical cells with a periodic boundary condition. This study have therefore simulated the microscopic system on a regular two-dimensional lattice where a fraction $\mathrm{p}$ of links are rewired at random. Each cell 'ij' is interacted to its neighbors in Moore local area. The random rewiring probability $\mathrm{p}$ (i.e., fraction $\mathrm{p}$ ) is always small enough, So it takes effect when Moore neighborhood radius is big enough. This study will discuss the influence of the rewiring probability $\mathrm{p}$ of the directed small-world network on dynamical behavior in different neighborhood.

\section{Fuzzy Model for Evolution of Online Public Opinion}

In the study of the Evolution of online public opinion, our model assumes the sentiment of the current netizen would be influenced by others around. The cells states in the grid are updated according to the local dynamics rules of each cell. Four essential characters need to be defined to set up a simulation run, the characters are the following:

- Self Emotional Tendencies of current netizen, it described sentiment polarity of current netizen to some events. Continuous opinion model is adopted in this paper.
- Self-Confidence of current netizen, the higher degree of self-confidence, the more difficultly influenced by those around it.

- The impact degree, which mean the affect of the netizen in the local area of current netizen, also, higher is the impact degree, the more influence will taken over sentimental Tendencies of current netizen.

- Sentiment orientation of other netizens around the current netizen.

Fuzzy set theory is a mathematical tool to model imprecise information and knowledge. In practice, precise values of all above characters are uncertain. Fuzzy rule-based systems (FRBS) is an appropriate approach to address the extent of the characters. The input variables of the FRBS are the characters above. The output variable is the new self Emotional Tendencies of current netizen. The input and output variables are linguistic variables, denoted as SET, SC, ID, SOON and NSET, respectively. Self Emotional Tendencies of current netizen (SET), the new self Emotional Tendencies of current netizen(NSET), and Sentiment orientation of other netizens around the current netizen (SOON) assume the following linguistic values oppose, disagree, neutral, agree, favor and self-confidence of current netizen (SC) and the impact degree (ID) adopt the linguistic values low,mid,high. The membership functions specify the meaning of the linguistic variables, as depicted in Fig.2-3 for SET, SC, ID, SOON and NSET, respectively. Now, each one of such adjectives like "oppose", "disagree" etc, is modelled mathematically by a fuzzy set through its membership function. This paper have chosen the gauss and triangular type for the membership functions.

The concept of Emotional Tendency of netizen has very rich and varied content, with fuzzy attribute, this uncertainty measurement always be inducted from Gaussian Model [30]. Therefore, to measure of the emotional tendencies in continuous opinion model, five level attitude is concerned: oppose, disagree, neutral, agree, favor. Each level correspond a fuzzy membership function. With the support of some experts from the Psychology area, Gauss Function is selected. The membership functions of SET, SOON and NSET demonstrated in Fig.2. 


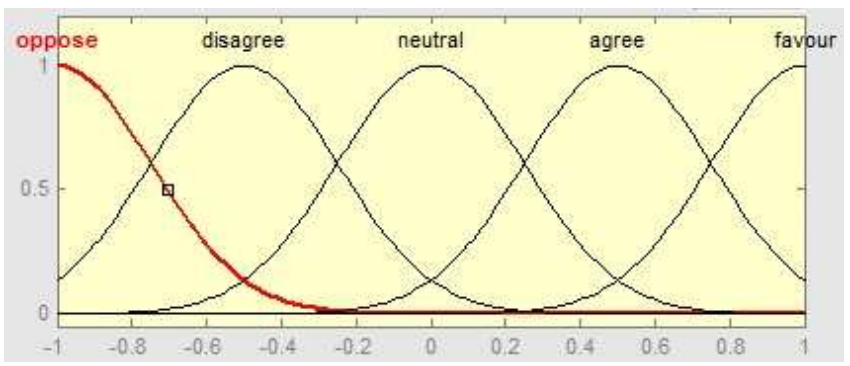

Fig. 2: The membership function: Self Emotional Tendencies of current netizen(SET); Sentiment orientation of other netizens around (SOON); The new self Emotional Tendencies of current netizen(NSET)

$$
\mu_{A}(x)=\operatorname{gaussm}_{A}\left(x, \sigma_{A}, a_{A}\right)=\exp \left[\frac{-\left(x-a_{A}\right)^{2}}{2 \sigma_{A}^{2}}\right]
$$

$A \in\{-1,-0.5,+0.5,+1\}, a_{A}, \sigma_{A}$ are the expected value and the standard deviation of the membership function. $\sigma_{A}=0.5, x \in[-1,1]$.

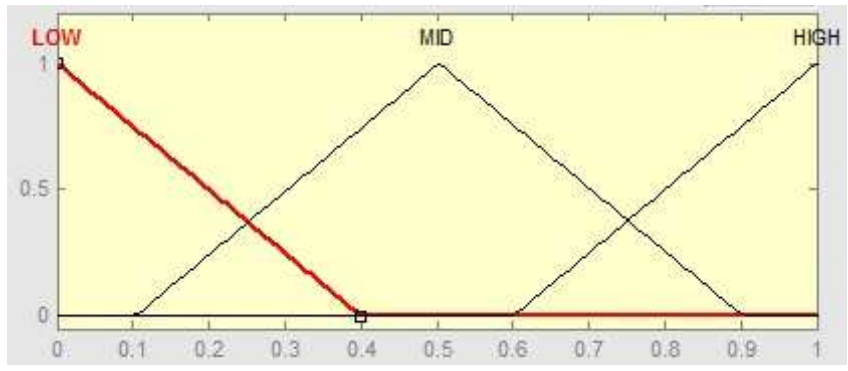

Fig. 3: The membership function: self-confidence of current netizen(SC); the impact degree(ID)

Self confidence of current netizen and the impact degree from other netizen is conducted with the triangular function to define ambiguity. Evaluating the netizens confidence and impact degree also can be represented by linguistic terms, High(high), medium(mid), low(low).

$$
\mu_{A}(x)=\left\{\begin{array}{cc}
0 & , \quad x<a_{1} \\
\left(x-a_{1}\right) /\left(a_{2}-a_{1}\right) & , a_{1} \leq x<a_{2} \\
\left(a_{3}-x\right) /\left(a_{3}-a_{2}\right) & , a_{2} \leq x<a_{3} \\
0 & , x \geq a_{3}
\end{array}\right.
$$

Here, Left point $\left(a_{1}\right)$, central point $\left(a_{2}\right)$ and right point $\left(a_{3}\right)$ determine the shape of membership, The membership functions of SC and ID demonstrated in Fig.3. $\left|a_{2}-a_{1}\right|=\left|a_{3}-a_{2}\right|=0.4$.

On the scheme of Fig.4, the knowledge base is translated by a fuzzy rule set and a fuzzy inference method. The number of fuzzy rules depend on the number changes in input and state variables. The number of combined fuzzy rules is $C_{5}^{1} \times C_{5}^{1} \times C_{3}^{1} \times C_{3}^{1}=255$. With the support of some experts from the psychology area, especially opinion psychologists, this study have formulated 70 fuzzy rules, such as:
1. If (SET is oppose) and (SC is HIGH) then (NSET is oppose)

2. If (SET is disagree) and (SC is HIGH) then (NSET is disagree)

3. If (SET is neutral) and (SC is HIGH) then (NSET is NEUTRAL)

4. If (SET is agree) and (SC is HIGH) then (NSET is AGREE)

5. If (SET is favour) and (SC is HIGH) then (NSET is FAVOUR)

6. If (SET is oppose) and (SC is MID) and (ID is HIGH) and (SOON is OPPOSE) then (NSET is oppose)

7. If (SET is disagree) and (SC is MID) and (ID is $\mathrm{HIGH}$ ) and (SOON is OPPOSE) then (NSET is oppose)

8. If (SET is neutral) and (SC is MID) and (ID is HIGH) and (SOON is OPPOSE) then (NSET is disagree)

9. If (SET is agree) and (SC is MID) and (ID is HIGH) and (SOON is OPPOSE) then (NSET is NEUTRAL)

10 .If (SET is favour) and (SC is MID) and (ID is HIGH) and (SOON is OPPOSE) then (NSET is AGREE)

11. If (SET is oppose) and (SC is MID) and (ID is MID) and (SOON is OPPOSE) then (NSET is oppose)

12. If (SET is disagree) and (SC is MID) and (ID is $\mathrm{MID})$ and (SOON is OPPOSE) then (NSET is oppose)

$$
\text { ....... }
$$

69. If (SET is agree) and (SC is LOW) and (ID is LOW) and (SOON is FAVOUR) then (NSET is FAVOUR)

70. If (SET is favour) and (SC is LOW) and (ID is LOW) and (SOON is FAVOUR) then (NSET is FAVOUR)

Some input parameter is ignored in the fuzzy rules. Such as rule 1 , the impact degree and Sentiment orientation from other netizens will have no effect on output, when the selfconfidence of current netizen is high enough.

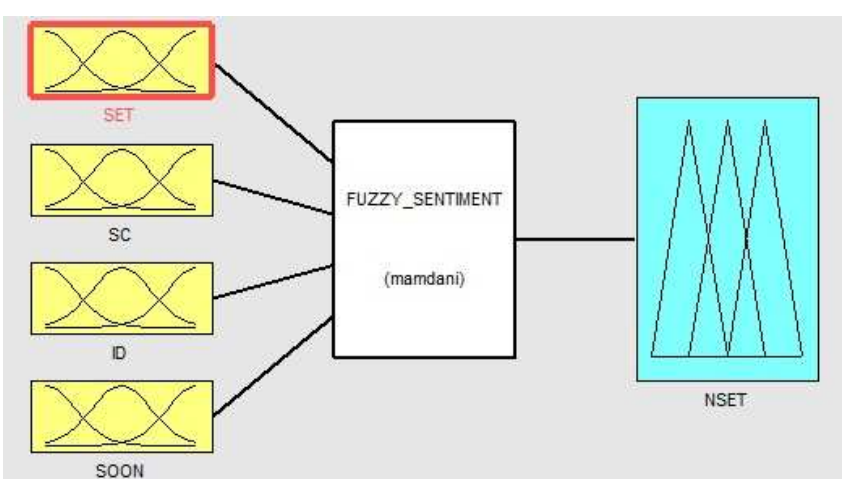

Fig. 4: The fuzzy sentiment inference system

From Mamdani inference method and defuzzification of the center-of-gravity, the system have obtained the new self Emotional Tendencies of current netizen (NSET) given by Fig.4, fuzzy sentiment inference system. 


\section{Simulation Results}

Computer simulations were carry out with varied parameters in order to investigate the influence of individual Tendencies to the evolution process. In the simulations, individuals change their opinions asynchronously. As far as we are concerned, in an update a Emotional Tendencies and one of its neighbors are picked at random to participate in the interaction, and a time step consists of $\mathrm{N}$ such update Emotional Tendencies of current netizen for a $\mathrm{N}$-size system.

The hot issues case at the beginning of 2012 is selected, China detonated its first social topic; Best-selling China author Han Han is suing anti-fraud crusader Fang Zhouzi, who recently accused Han of hiring ghostwriters for most of his works. Han and his lawyer were preparing for the lawsuit. Which one do you support, Hanhan or Fangzhouzi? Some initial data come from the blog of Hanhan (http://blog.sina.com.cn /twocold) and the microblog of Fangzhouzi (http://weibo. com/fangzhouzi). And the initial state of some typical netizen is set by Table 1. By continuous opinion model, the value of excessively opposition is set to 1 , and the decimal between 0 and 1 show the value of disagreement, and vice versa. A portion of initial data come from the weblog of the two parties. Corresponding state variables are identified by the condition of speech and expression in web, and the impact degree come from the follower count and the number of forum posts. Also the location of these agents is simulated by network environment. The portion of the initial data is shown in Table 1. To understand how the public opinion of the system along one of the two competing opinions is balanced, it is supposed that the initial density of two opinion is nearly same as another, and original opinions are fully mixed. The strategies listed above are defined as follows. $A_{n_{1}}$ is the set of supporter, $n_{1}$ is the count of set, $S E T_{i \in A_{n_{1}}} \in[0.1,0.9]$. $A_{n_{2}}$ is the set of opposition tend, $n_{2}$ is the count of it, $S E T_{i \in A_{n_{2}}} \in[-0.9,-0.1] . A_{n_{3}}$ is the set of the centre, $n_{3}$ is the number of it, $S E T_{i \in A_{n_{3}}} \in[-0.1,0.1]$. Lattice space is set $20 \times 20$, and $n_{1}: n_{2}: n_{3}=1: 1: 0.25$. The initial state of lattice space is shown by left plot of Fig.5.

Table 1: The Initial Data Set

\begin{tabular}{ccccc}
\hline Individual netizen & SET & SC & ID & Cell position \\
\hline$N_{1}$ & -1 & 1 & 0.8 & 2,12 \\
$N_{2}$ & 1 & 1 & 0.8 & 16,3 \\
$N_{3}$ & 1 & 1 & 0.5 & 18,9 \\
$N_{4}$ & -0.7 & 0.7 & 0.4 & 2,2 \\
$N_{5}$ & -0.4 & 0.6 & 0.4 & 3,7 \\
$N_{6}$ & -0.2 & 0.4 & 0.4 & 5,9 \\
$N_{7}$ & -0.5 & 0.5 & 0.4 & 5,17 \\
$N_{8}$ & 0.5 & 0.5 & 0.4 & 17,5 \\
$N_{9}$ & 0.6 & 0.4 & 0.5 & 19,9 \\
\hline
\end{tabular}

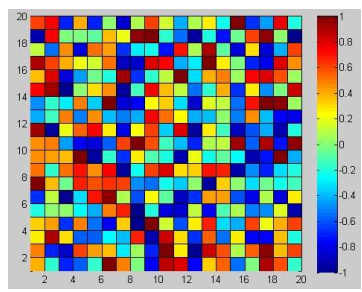

(a)

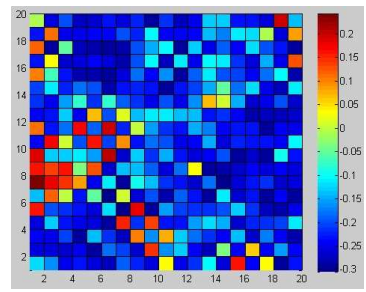

(b)
Fig. 5: Dimensional configuration plots of opinions Fig.5(a) and agents' tendency Fig.5(b) when time step is 100 , agent number $N=400$, Moore neighborhood with radius $r=1$; The random rewiring probability $p=$ 0.5 , color illustration on the right represent the value of Emotional Tendency

The simulation results reveal the relation between individual tendencies and opinions. Fig.5 shows the dimensional configurations of Tendencies and opinions in a typical run at time step 100 with the topology of two-dimensional lattices. According to the plot, the shape of domains in the opinion configuration is similar to that in the inclination configuration. Agents which keep the preponderant state attain an intense inclination more easily, and this trend comes from the difference of original densities between two opinions. If netizens increase the concentration of a certain opinion, tendencies for this opinion will grow at a faster rate.

According to the evolution with neighborhood radius and the small world of network $\mathrm{R}$ random rewiring probability $P$ value, the combination simulation experiments is carried out when $R=1, R=5$, and $P=0, P=0.1, P=0.3, P=0.5$. Compared Figures in Fig.6, Emotional Tendencies for winning opinion will grow at a faster rate if larger radius and random rewiring probability are taken. Also the clash of opinion will always come down. Through the analysis of the emulation results, he opinion concentration polarization, are revealed by Fig.6. It is reflected that wider is the diffusion process range of online public opinion spread, the more quick is the convergence speed.

This study also calculated the average emotional tendency value of all agents in different evolution steps, which can illustrate the change of public opinion. Fig.7 shows that the average emotional tendency in evolution process present convergence condition. Different arguments or parameters will have an effect on the speed of convergence. The convergence rate is slow where $R=1, P=0$, and the speed of convergence is fast where $R=1, P=0.5$. It shows that the speed of dissemination of online public opinion is slow when netizens limited in a quite narrow local area. If they can accept information from outside, the speed of evolution is quiet fast from part to whole. 


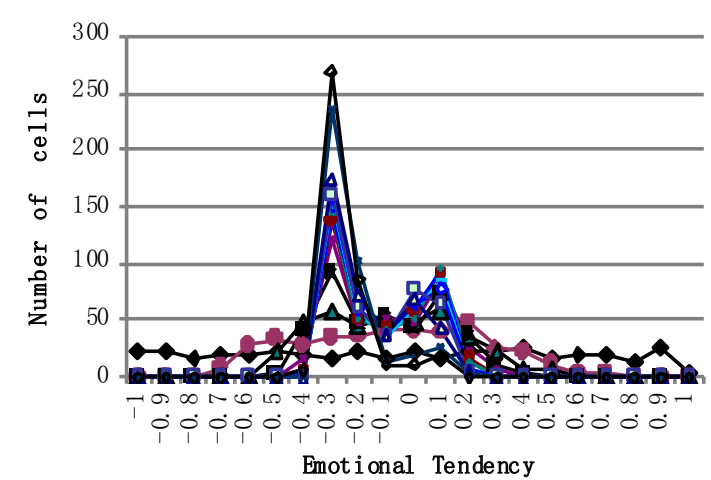

(a) $\mathrm{R}=1, \mathrm{P}=0$

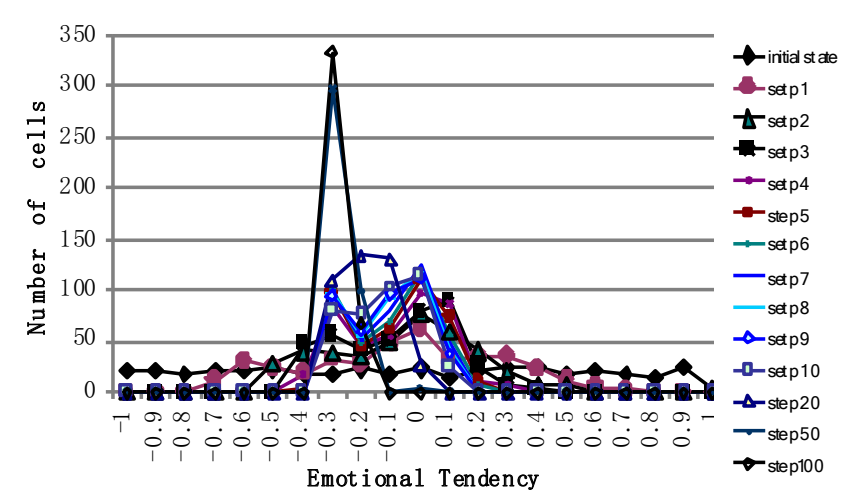

(b) $\mathrm{R}=5, \mathrm{P}=0$

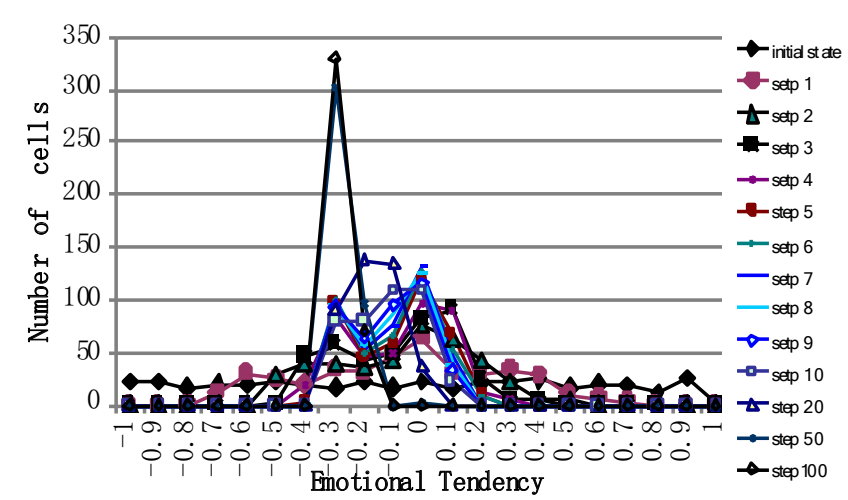

(c) $\mathrm{R}=5, \mathrm{P}=0.1$

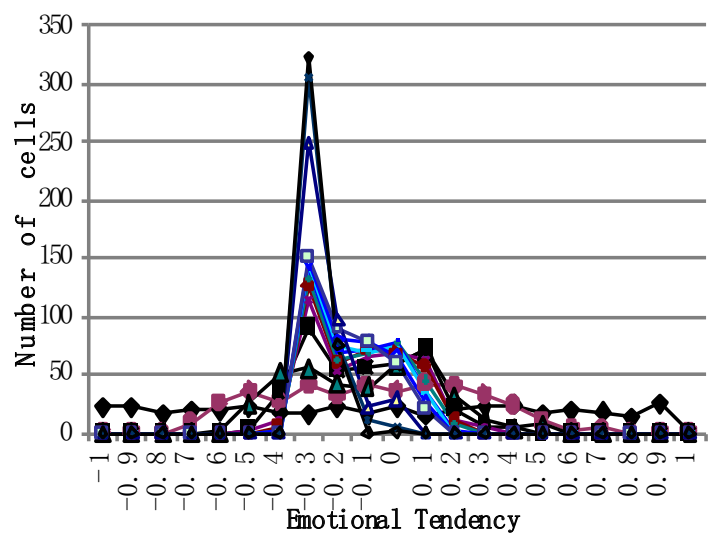

(d) $\mathrm{R}=1, \mathrm{P}=0.3$

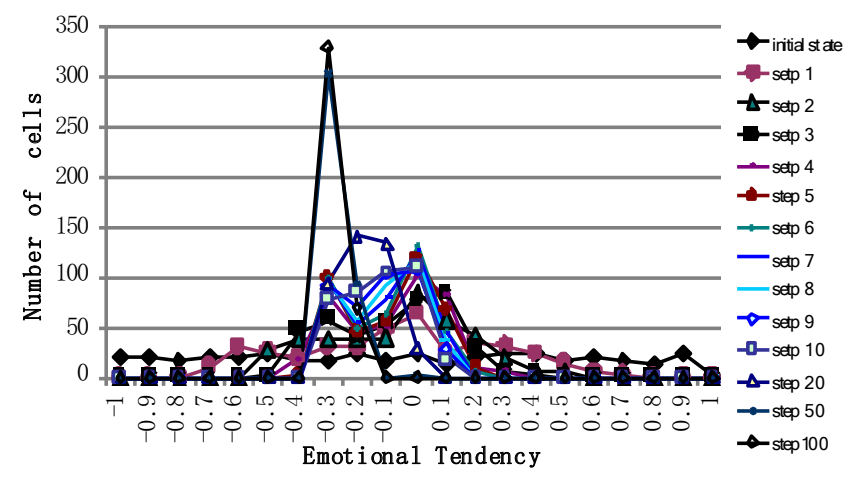

(e) $\mathrm{R}=5, \mathrm{P}=0.3$

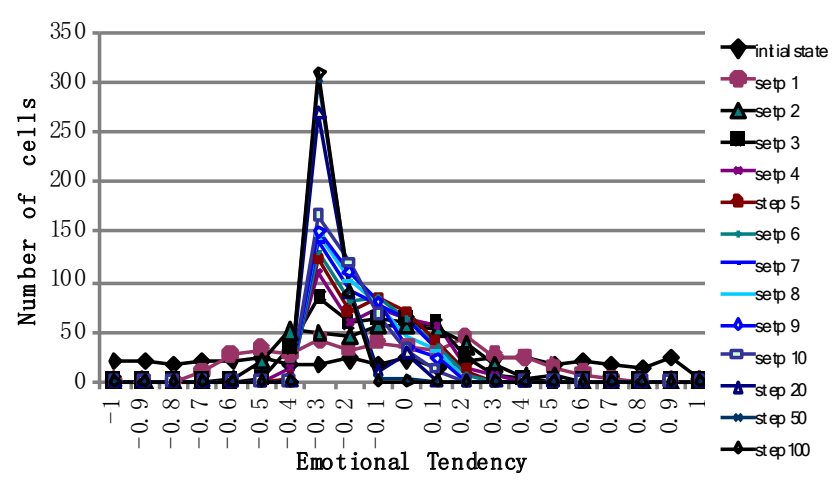

(f) $\mathrm{R}=1, \mathrm{P}=0.5$

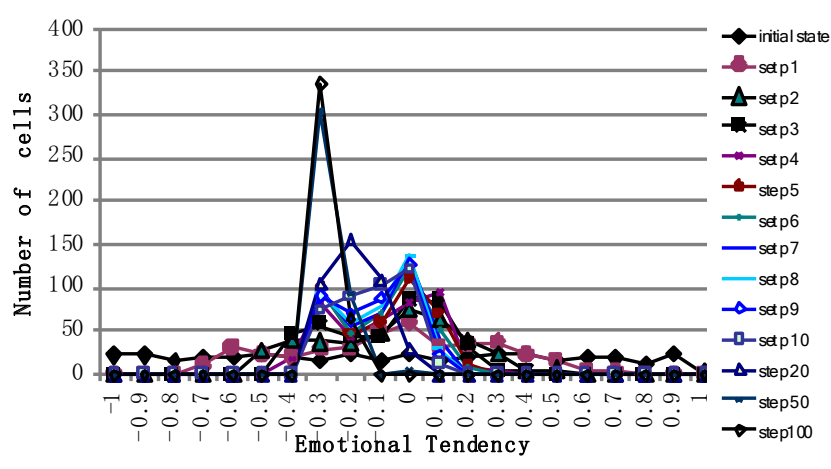

(g) $\mathrm{R}=5, \mathrm{P}=0.5$

Fig. 6: Fig.6(a)-Fig.6(g): Agents' tendency Scatter diagrams with various Moore neighborhood radius and different random rewiring probability. The number of agents labels the vertical axis of the above plot. Emotional tendency marks the horizontal axis with the different parameters

\section{Conclusion}

The contributions of this paper include a formal modeling and analysis approach for the Evolution of Online Public Opinion based on Fuzzy cellular automata rules in directed world networks. Through the depiction mentioned above, Emotional Tendencies of netizens are 


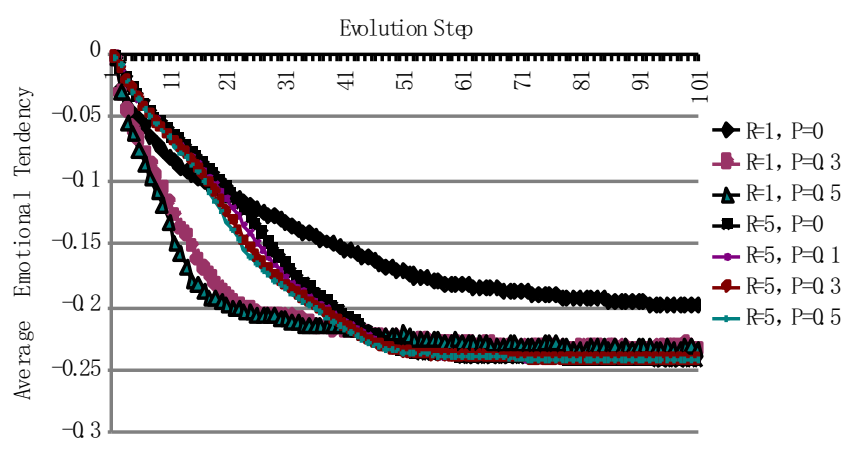

Fig. 7: Average emotional tendency evolution with various radius and different random rewiring probability

linguistic terms, so do other essential characters. The rule-based fuzzy system can simulate more exactly. Through the analysis of the simulation results, the opinion concentration, polarization, are revealed. The results of simulation also show that all four essential characters have a certain influence on individual behavior as well as group's. And the changing character of neighbors will influence individual behavior. Simulation results also indicate that the model can better fit with the public opinion evolution laws.

\section{Acknowledgement}

This work is supported by National Social Science Foundation of China (No.12BTQ055), National Natural Science Foundation of China (No.71071114), Foundation of Zhejiang Educational Committee (No. Y201224457). We also wish to thank the reviewers for their very useful comments and suggestions.

\section{References}

[1] http://www.cnnic.net.cn.

[2] http://en.wikipedia.org/wiki/Public\{_\}opinion.

[3] Zhang, M., Ye, X, A Generation Model to Unify Topic Relevance and Lexicon-based Sentiment for Opinion Retrieval, In: Proceedings of SIGIR, 411-418 (2008).

[4] Zhang, W., Yu, C., Meng, W., pinion Retrieval from Blogs In Proceedings of CIKM, 831-840 (2007).

[5] Li, F., Tang, Y., Huang, M., Zhu, X., swering Opinion Questions with Random Walkson Graphs ,In Proceedings of ACL, 737-745 (2009).

[6] G. Toscani, Kinetic models of opinion formation ,communications in mathematical sciences, 3, 481-496 (2006).

[7] S. Galam, Contrarian deterministic effects on opinion dynamics:" the hung elections scenario, physica A. 333, 453460 (2004).

[8] S. Galam, Real space renormalization group and totalitarian paradox of majority rule voting, physica A. 285, 66-76 (2000).
[9] G. Weisbuch, G. Deffuant, F. Amblard, Persuasion dynamics, physica a-statistical mechanics and its applications, 353, 555575 (2005).

[10] Amblard F, Deffuant G, The role of network topology on extremism propagation with the relative agreement opinion dynamics, physica a-statistical mechanics and its applications, 343, 725-738 (2004).

[11] R. Hegselmann and U. Krause, J. Truth and Cognitive Division of Labour First Steps towards a Computer Aided Social Epistemology, the journal of artificial societies and social simulation, 3, 361-388 (2006).

[12] Alves, S. G., Oliveira Neto, N. M. and Martins, M. L., Electoral surveys' influence on the voting processes: a cellular automata model ,Physica A, 1, 601-614 (2002).

[13] Weisbuch, G. and Stauffer, D., Adjustment and social choice, Physica A, 323, 651-662 (2003).

[14] Breckling, B., Mller, F., Reuter, H., Holker, F., Franzle, O., Emergent properties in individual-based ecological modelsintroducing case studies in an ecosystem research context. ecological modeling, 4, 376-388 (2005).

[15] von Neumann J. Papers of John von Neumann on Computing and Computer Theory in Reprint Series on the History of Computing. Boston: MIT Press, (1963).

[16] Wolfram S. Theory and Applications of Cellular Automata in: Advanced Series on Complex Systems. Singapore: World Science, (1986).

[17] Seck-Tuoh-Mora Juan C., Martinez Genaro J., Alonso-Sanz Ramon, Invertible behavior in elementary cellular automata with memory ,information sciences, 199, 125-132 (2012) .

[18] Betel Heather, Flocchini Paola, On the relationship between fuzzy and Boolean cellular automata, theoretical computer science, 8, 703-713 (2011).

[19] Zadeh, L. A., Fuzzy sets. Information and Control, 3, 338353 (1965).

[20] L. C. Barros, M. B. Leite, R. C. Bassanezi, The SI epidemiological models with a fuzzy transmission parameter, Computers and Mathematics with Applications, 11, 16191628 (2003).

[21] N. Ortega, L. C. Barros, E. Massad, Fuzzy gradual rules in epidemiology, Kybernetes: The International Journal of Systems and Cybernetics, 4, 460-477 (2003).

[22] R. M. Jafelice, L. C. Barros, R. C. Bassanezi, F. Gomide, Fuzzy modeling in asymptomatic HIV virus infected population, Bulletin of Mathematical Biology, 6, 1597-1620 (2004).

[23] R. M. Jafelice, L. C. Barros, R. C. Bassanezi, F. Gomide, Methodology to determine the evolution of asymptomatic HIV population using fuzzy set theory,International Journal of Uncertainty, Fuzziness and Knowledge-Based Systems, 13, 39-58 (2005).

[24] Park Keon-Jun; Oh Sung-Kwun; Kim Yong-KabDesign of Interval Type-2 Fuzzy Set-based Fuzzy Neural Networks and Its Optimization Using Generation-based Evolution, information-an international interdisciplinary journal, 5, 1775-1790 (2011).

[25] Liu Yan, Modelling sustainable urban growth in a rapidly urbanising region using a fuzzy-constrained cellular automata approach, international journal of geographical information science, 26, 151-167 (2012).

[26] Tang Junmei,Modeling urban landscape dynamics using subpixel fractions and fuzzy cellular automata, environment and planning b-planning \& design, 38, 903-920 (2011). 
[27] R. Motta Jafelice,, B. F. Z. Bechara , L. C. Barros , R. C. Bassanezi, F. Gomide, Cellular automata with fuzzy parameters in microscopic study of positive HIV individuals. Mathematical and Computer Modelling, 50, 32-44 (2009).

[28] Albert R and Barabasi A L, Statistical mechanics of complex networks, reviews of modern physics, 74, 47-97 (2002).

[29] Watts D J and Strogatz S HCollective dynamics of 'smallworld' networks, Nature, 393, 440-442 (1998).

[30] Shi Fuqian, Employing Emotional Cellular Models and its Similarity Computing in Valence - Arousal based Semantic Inference, information-an international interdisciplinary journal,, 15, 467-482 (2012).

[31] K. K. Gupta1, R. Gupta, Wavelet Based Speckle Filtering of the SAR Images, International Review on Computers and Software, 1, 224-232 (2006).

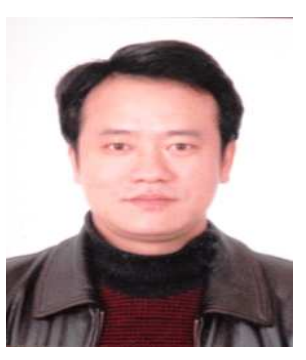

Tao Chen received the B.S. degree in applied mathematics from Ningbo University in 1994, and the M.S. degree in computer science and technology from Tsinghua University in 2004. He is currently working toward the Ph.D. degree in information management and system at Tongji University. $\mathrm{He}$ is also an Associate Professor in Department of information management, Ningbo University. His research interests are in the areas of sentiment analysis and opinion mining.

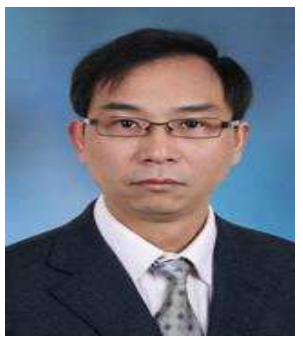

Jie Lin is the professor and doctoral tutor in Department of Management Science and Engineering, School of Economics and Management, Tongji University. $\mathrm{He}$ received his B.E. degree in Electronic Engineering from University of Electronic Science and Technology of China, in 1989 and the M.S. and $\mathrm{Ph} . \mathrm{D}$ degrees from Southwest Jiaotong University in 1995 and 1999. He was working in Princeton University as an Advanced Visiting Scholar in 2009. His research interests are in the areas of decision support system and multi-agent simulation. 